\title{
Development of an Enzyme Immunoassay Specific for a Core Protein Epitope of a Novel Small Basement Membrane Associated Heparan Sulphate Proteoglycan from Human Kidney ${ }^{1}$ )
}

\author{
Georg Stöcker, Elmar Stickeler, Silke Switalla, Dagmar-Christiane Fischer, Helmut Greiling and \\ Hans-Dieter Haubeck
}

Institute for Clinical Chemistry and Pathobiochemistry, RWTH Aachen, Aachen, Germany

\begin{abstract}
Summary: Heparan sulphate proteoglycans are major components of the glomerular basement membrane and play a key role in their molecular organization and function. Moreover, their presence is essential for the maintenance of the selective permeability of the glomerular basement membrane. Recently, we have isolated and characterized a novel, small basement membrane associated heparan sulphate proteoglycan from human aorta and kidney. Using specific monoclonal antibodies we have shown that the novel heparan sulphate proteoglycan is predominantly located in the glomerular basement membrane, to a lesser extent in the basement membrane of tubuli, and also in the mesangium. Turnover or, in the course of kidney diseases, degradation of heparan sulphate proteoglycan from glomerular basement membranes may lead to urinary excretion of heparan sulphate proteoglycan. Therefore, changes in the structure and function of glomerular basement membranes may be directly detected by measuring the excretion of a component of this basement menbrane, e. g. heparan sulphate proteoglycan into urine. Here we describe the establishment of an enzyme immunoassay for the sensitive detection of the novel, small heparan sulphate proteoglycan in urine. In this assay the specific monoclonal antibody $1 \mathrm{~F} 10 / \mathrm{B} 8$, which recognizes a core protein epitope, was used to detect the polyanionic heparan sulphate proteoglycan bound to the surface of a cationic charge modified microtitre plate. This assay allows the sensitive and specific detection of the small heparan sulphate proteoglycan, which is released from the glomerular basement membrane into urine during normal turnover and also in the course of kidney diseases.
\end{abstract}

\section{Introduction}

Heparan sulphate proteoglycans are major components of the glomerular basement membranes and play a key role in their molecular organization and function (reviewed in 1.c. $(1-3))$. The presence of heparan sulphate proteoglycans is essential for the maintenance of the selective permeability of the glomerular basement membrane $(3-5)$. Loss of the anionic sites provided by the heparan sulphate proteoglycans is associated with proteinuria and has been observed in a number of nephropathies $(3-10)$. Such a loss might occur as the result of reduced synthesis, enhanced turnover, or degradation of heparan sulphate proteoglycans. However, the exact mechanism of this process is not well understood.

Recently, we have isolated and characterized a novel, small basement membrane associated heparan sulphate proteoglycan from human aorta and kidney. Using specific monoclonal antibodies we have shown that this heparan sulphate proteoglycan is predominantly located in the glomerular basement membrane, and to a lesser extent in the basement membrane of tubuli and also in

1) This work was supported by grant $\mathrm{Nr}$. Ha 1565/3-1 from the Deutsche Forschungsgemeinschaft (DFG), Bonn the mesangium. Partial amino acid sequence data clearly show that this heparan sulphate proteoglycan is distinct from the large basement membrane associated heparan sulphate proteoglycan (perlecan) (11).

Measurement of heparan sulphate proteoglycans, released from the glomerular basement membrane during normal turnover and also in the course of kidney diseases and excreted into urine, might be a sensitive marker for the detection of alterations of the glomerular basement membrane. In the present study we describe the development of an enzyme immunoassay, using the specific monoclonal antibody $1 \mathrm{~F} 10 / \mathrm{B} 8$, which recognizes a core protein epitope of the small heparan sulphate proteoglycan, which allows the sensitive detection of the novel small heparan sulphate proteoglycan in urine.

\section{Materials and Methods}

Reagents

Sephadex G-25, Sepharose Q, Protein-G-Sepharose, TSK G 4000 SW column $(7.5 \mathrm{~mm} \times 300 \mathrm{~mm})$, TSK G SWP guard-column $(7.5$ $\mathrm{mm} \times 75 \mathrm{~mm})$ and preformed gels for SDS poly-acrylamide gel electrophoresis (4-15\% gradient) were obtained from Pharmacia/ LKB (Freiburg, Germany). Hydroxylapatite and Zeta-Probe blotting membrane were obtained from Bio-Rad (Munich, Germany). Polyvinylidenfluoride blotting membranes (Fluorotrans) were 
purchased from Pall (Dreieich, Germany). High molecular mass standards, 3-[3-(cholamido-propyl)-dimethylamino]-1-propanesulphonate (CHAPS), benzamidine hydrochloride, 6-amino-hexanoic acid, phenylmethylsulphonyl fluoride and $\mathrm{N}$-ethylmaleinimide were purchased from Sigma (Deisenhofen, Germany). Diaminobenzamidine-tetrahydrochloride was from Serva (Heidelberg, Germany), $o$-phenylenediamine and peroxidase-conjugated rabbit-antimouse-Ig-antiserum were from DAKO (Kopenhagen, Denmark) and peroxidase-conjugated goat-antiserum against mouse-Ig were from Dianova (Hamburg, Germany). Heparinase (heparin lyase; EC 4.2.2.7) ${ }^{2}$ ) and heparitinase (heparan sulfate lyase EC 4.2.2.8 ${ }^{2}$ ) were obtained from Seikagaku (Tokio, Japan). Sulphosuccinimidyl-6-(biotinamido)-hexanoate (NHS-LC-biotin) was from Pierce (Munich, Germany). Polyethylene glycol 1500 (PEG 1500) and trypsin (EC 3.4.21.4) were from Boehringer Mannheim (Mannheim, Germany). Dulbecco's modified Eagle's medium was from GIBCO, BRL (Eggenstein, Germany). Casein was from Sigma (Munich, Germany). All remaining reagents were of analytical grade (Merck, Darmstadt, Germany).

\section{Methods \\ Small basement membrane associated heparan sulphate proteoglycan from human aorta and kidney}

The novel, small heparan sulphate proteoglycan was isolated from human aorta and kidney and characterized biochemically and immunochemically as described elsewhere $(11-13)$. Briefly: Proteoglycans were extracted by $4 \mathrm{~mol} / 1$ guanidine hydrochloride, 0.05 $\mathrm{mol} / \mathrm{l}$ sodium acetetate, $(\mathrm{pH} 5.8)$ containing $5 \mathrm{~g} / 1$ CHAPS and the protease inhibitors benzamidine hydrochloride $(0.005 \mathrm{~mol} / \mathrm{l})$, 6 aminohexanoic acid $(0.1 \mathrm{~mol} / \mathrm{l})$, phenylmethylsulphonyl fluoride $(0.005 \mathrm{~mol} / \mathrm{l}), \mathrm{N}$-ethylmaleimide $(0.01 \mathrm{~mol} / \mathrm{l})$, and EDTA $(0.05$ $\mathrm{mol} / \mathrm{l}$ ). The $4 \mathrm{~mol} / \mathrm{l}$ guanidine hydrochloride extract was equilibrated with $0.05 \mathrm{~mol} / 1$ Tris, $0.05 \mathrm{~mol} / 1$ sodium acetate buffer $(\mathrm{pH}$ 5.8) containing $7 \mathrm{~mol} / 1$ urea, $0.05 \mathrm{~mol} / 1 \mathrm{NaCl}, 0.05 \mathrm{~mol} / 1 \mathrm{LiCl}, 2 \mathrm{~g} / 1$ CHAPS and protease-inhibitors $(0.001 \mathrm{~mol} / 1 \mathrm{~N}$-ethylmaleimide, $0.002 \mathrm{~mol} / 1$ benzamidine hydrochloride and $0.001 \mathrm{~mol} / \mathrm{l}$ phenylmethylsulphonyl fluoride) by size-exclusion chromatography on Sephadex G-25. Heparan sulphate proteoglycan was isolated by anion-exchange chromatography on Sepharose Q (Pharmacia/LKB, Freiburg, Germany) and further purified by hydroxylapatite chromatography. Heparan sulphate proteoglycan was characterized by SDS-PAGE prior to and after digestion with glycosaminoglycanspecific enzymes. For immunoblots the small basement membrane associated heparan sulphate proteoglycan from human kidney was submitted to SDS-PAGE on preformed $4-15 \%$ gradient gels (Pharmacia Phast system, Pharmacia/LKB) and blotted to polyvinylidenfluoride membranes (Fluorotrans, Pall) by diffusion (13). After blocking of non-specific binding sites, heparan sulphate proteoglycan was detected by the specific monoclonal antibodies 1F10/B8 or 2E2/B5 and a peroxidase-conjugated goat-anti-mouseIgG antibody (Dianova) and 3,3'-diaminobenzidine tetra-hydrochloride as substrate.

Whereas heparan sulphate proteoglycan isolated from aorta revealed an $M_{\mathrm{r}}$ distribution of $>200000-80000$, for the small heparan sulphate proteoglycan from kidney an $M_{\mathrm{r}}$ distribution of 160000-30000 was found. The size of the core proteins was determined to be $M_{\mathrm{r}} 24000$ and $M_{\mathrm{r}} 22000$ for aorta and kidney, respectively. The monoclonal antibody 1 F10/B8 (see below) recognizes both core proteins. However, small heparan sulphate proteoglycans from the kidney and aorta can be discriminated according to their different size, which is mainly due to differences in their glycosaminoglycan chain moieties. The size of the heparan sulphate proteoglycan isolated from urine was in the $M_{\mathbf{r}}$ range of $160000-30000$, indicating that it is derived mainly from the kidney. Partial amino acid sequence data clearly show that this heparan sulphate proteoglycan is distinct from the large basement membrane associated heparan sulphate proteoglycan (perlecan).

\section{2) Enzymes}

Heparinase (heparin lyase EC 4.2.2.7)

Heparitinase (heparan sulfate lyase EC 4.2.2.8)
Cloning and sequencing of the core protein of this heparan sulphate proteoglycan are presently in progress.

\section{Monoclonal antibodies}

Monoclonal antibodies were raised in mice according to a modified protocol of the basic outline of Köhler and Milstein (14). Briefly: $100 \mu \mathrm{g}$ of the small basement membrane associated heparan sulphate proteoglycan in a volume of $300 \mu \mathrm{l}$ were mixed with an equal volume of Freund's complete adjuvant and injected intraperitoneally and subcutaneously into 8 week old BALB/c female mice. After 6 weeks the mice received booster injections of the antigen in Freund's incomplete adjuvant and a final injection 3 days before fusion. Serum titers of pre-immune and immune sera were determined with a semiquantitative slot blot enzyme immunoassay (see below). Spleen cells of the mouse with the highest serum titre were fused with the BALB/c myeloma X63-Ag8/653 with polyethylene glycol. Cells were plated in 96-well plates in Dulbeccos's modified Eagle's medium containing foetal calf serum, volume fraction 0.1 and hypoxanthine-aminopterine-thymidine mixture. Hybridoma supernatants were tested by the slot blot enzyme immunoassay. Hybridomas showing a positive reaction were cloned and subcloned by limiting dilution.

\section{Screening and characterization of monoclonal antibodies}

Supernatants were tested for specific antibody against the small basement membrane associated heparan sulphate proteoglycan using a slot blot enzyme immunoassay on a Zeta Probe membrane (BioRad). Briefly: $50 \mathrm{ng}$ per slot of the small basement membrane associated heparan sulphate proteoglycan preparation were incubated for $10 \mathrm{~min}$ at room temperature on a Zeta Probe membrane. After blocking of the membrane with BLOTTO $(20 \mathrm{~g} / 1$ dry milk powder in phosphate-buffered saline) for $2 \mathrm{~h}, 200 \mu \mathrm{l}$ of hybridoma supernatants were incubated for $4 \mathrm{~h}$ at room temperature. Bound antibodies were detected by a peroxidase-conjugated second antibody from goat and diaminobenzidine $/ \mathrm{H}_{2} \mathrm{O}_{2}$ as substrate. Subclass determination of the specific monoclonal antibodies was done by an enzyme immunoassay using subclass specific antisera (Dianova, Hamburg, Germany). Purification of monoclonal antibodies from cell culture supernatants was done by protein $\mathrm{G}$ affinity chromatography according to the manufacturers instructions (Pharmacia/LKB).

\section{Heparan sulphate proteoglycan enzyme immunoassay}

Using one of the monoclonal antibodies (1F10/B8) against the small heparan sulphate proteoglycan from human aorta, a sensitive enzyme immunoassay for heparan sulphate proteoglycan was developed. In principle in this assay the polyanionic heparan sulphate proteoglycan from the sample is bound on a cationic charge modified 96-well microtitre plate (Greiner, Nürtingen, Germany)

Antigen-containing samples $(100 \mu 1 /$ well $)$ diluted $1: 10$ in 0.05 $\mathrm{mol} / \mathrm{l}$ sodium phosphate buffer $(\mathrm{pH} 4.5)$, containing $0.5 \mathrm{~g} / 1$ Tween 20 were incubated on a cationic charge modified 96-well microtitre plate (kindly provided by Greiner, Nürtingen, Germany) for $60 \mathrm{~min}$ at room temperature. After washing with $0.1 \mathrm{~mol} / 1 \mathrm{KH}_{2} \mathrm{PO}_{4} /$ $\mathrm{K}_{2} \mathrm{HPO}_{4}$ (pH 6.5), non-specific binding sites were blocked by 10 $\mathrm{g} / 1$ casein (Sigma) in phosphate-buffered saline $(200 \mu \mathrm{l} / \mathrm{well}, 60$ $\mathrm{min}, 37^{\circ} \mathrm{C}$. All incubations were carried out in a humidified atmosphere. Bound heparan sulphate proteoglycan was detected by the heparan sulphate proteoglycan specific monoclonal antibody $1 F 10 /$ B8. The specific antibody $(100 \mu \mathrm{l} /$ well $)$, diluted appropriately in blocking solution, was allowed to bind for $120 \mathrm{~min}$ at room temperature. To remove unbound antibody, wells were washed 5 times ( $200 \mu \mathrm{l} /$ well of $0.1 \mathrm{~mol} / 1 \mathrm{KH}_{2} \mathrm{PO}_{4} / \mathrm{K}_{2} \mathrm{HPO}_{4}$ buffer, $\mathrm{pH} 6.5$ ). Bound antibody was detected by the use of a second peroxidase-labelled polyclonal goat anti-mouse antiserum (Dianova). Unbound second antibody was removed by washing 5 times with $0.1 \mathrm{~mol} / 1$ citrate/ citric acid $\mathrm{pH}$ 5.0.

Substrate solution $(100 \mu 1)$ was added to the wells $(8 \mathrm{mg} o$-phenylenediamine dissolved in $12 \mathrm{ml} 0.1 \mathrm{~mol} / 1$ citrate/citric acid pH 5.0 and $\left.5 \mu \mathrm{H}_{2} \mathrm{O}_{2}(300 \mathrm{~g} / \mathrm{kg})\right)$. Colour development was stopped by addition of $0.5 \mathrm{~mol} / 1 \mathrm{H}_{2} \mathrm{SO}_{4}(100 \mu \mathrm{l} /$ well $)$. The plates were read 
using an ELISA plate reader (SLT, Overath, Germany) at a wavelength of $492 \mathrm{~nm}$; absorbance at $690 \mathrm{~nm}$ was used for background subtraction.

For heparan sulphate proteoglycan excreted into urine, data were expressed either as $\mu \mathrm{g} / 1$ of heparan sulphate proteoglycan or as heparan sulphate proteoglycan/creatinine excretion ratio $(\mathrm{mg} / \mathrm{mol}$ creatinine). All samples were measured at least in duplicate. No inhibition of binding was observed in this assay when other polyanionic molecules such as heparin or keratan sulphate proteoglycans were added in control experiments.

\section{Results and Discussion}

Heparan sulphate proteoglycans are major components of the glomerular basement membranes and are essential for the maintenance of their selective permeability (reviewed in 1. c. $(1-5)$ ). Loss of the anionic sites provided by the heparan sulphate proteoglycans is associated with proteinuria and has been observed in a number of nephropathies $(3-10)$. Different types of heparan sulphate proteoglycans have been described in basement membranes $(1,2,15-18)$ but the involvement of these different heparan sulphate proteoglycans, and especially of the novel, small heparan sulphate proteoglycan in the glomerular selective permeability has not yet been clarified.

The aim of the present study was to develop an enzyme immunoassay which allows the sensitive detection of the small heparan sulphate proteoglycan excreted into urine and to analyze whether changes of the structure and function of glomerular basement membranes can be detected by measurement of the excretion of a component of this basement membrane, e.g. heparan sulphate proteoglycan, into urine. However, an important question was wether the heparan sulphate proteoglycan excreted into urine is derived only from the kidney. This question was addressed by comparison of the different heparan sulphate proteoglycans.

\section{Comparison of the small basement membrane} associated heparan sulphate proteoglycan isolated from human aorta and kidney and from urine

Heparan sulphate proteoglycans isolated from human aorta and kidney by several chromatographic steps and from a sample of urine (which was concentrated, but without further purification) were submitted to SDSPAGE on an $8-25 \%$ gradient gel and subsequently blotted onto a positively-charged nylon membrane. Immunochemical detection was performed using the heparan sulphate proteoglycan specific monoclonal antibody 1F10/B8. Figure 1 clearly shows that the relative molecular mass distribution of heparan sulphate proteoglycan from urine is comparable to that from human kidney, with an $M_{\mathrm{r}}$ of $160000-30000$. In contrast, the small heparan sulphate proteoglycan from aorta has an $M_{\mathrm{r}}$ distribution of $>200000-80000$, indicating that the heparan sulphate proteoglycan found in urine is derived from the kidney. Furthermore, the selective permeability of the intact glomerular basement membrane effectively presents the excretion of highly negatively charged molecules, such as heparan sulphate proteoglycan, from the aorta with an $M_{\mathrm{r}}$ distribution of $>200000-80000$. Therefore the excreted heparan sulphate proteoglycan in normal subjects is most likely released from the glomerular basement membrane and not from other sources, e. g. other basement membranes such as the aortal basement membrane. This is also supported by the failure to detect the large basement membrane associated heparan sulphate proteoglycan (perlecan) in the urine $(E$. Schleicher, personal communication).

Development of an enzyme immunoassay specific for a core protein epitope of the small basement membrane associated heparan sulphate proteoglycan from human kidney

Small heparan sulphate proteoglycan isolated from human aorta was used for immunization of mice. A series of more than 20 monoclonal antibodies was obtained. Two of these monoclonal antibodies (1F10/B8 and 2E2/ B5) were selected for further characterization and subsequently used for the development of the enzyme immunoassay. To determine the specificity of these antibodies, native and enzymatic deglycosylated heparan sulphate proteoglycan were submitted to SDS-PAGE and Western blotting. The epitopes, recognized by the monoclonal antibodies 1F10/B8 and 2E2/B5, were not influenced by enzymatic deglycosylation of the heparan sulphate proteoglycan (data not shown), indicating that

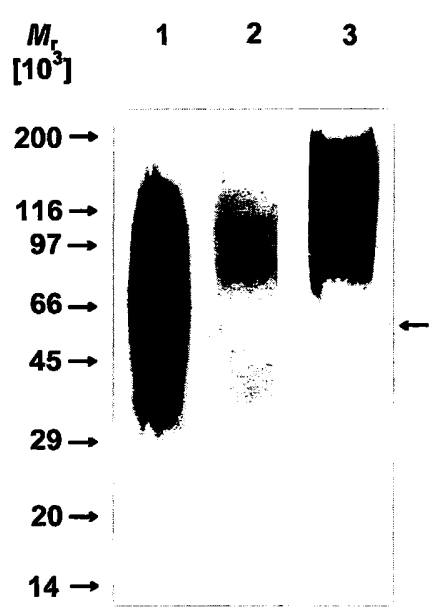

Fig. 1 Western blot of heparan sulphate proteoglycan from human aorta and kidney and from urine

Heparan sulphate proteoglycan isolated from human kidney (1), urine (2), and aorta (3) was submitted to SDS-PAGE on an 8-25\% gradient gel and subsequently blotted onto a positively charged nylon membrane. Immunochemical detection was performed using the monoclonal antibody 1F10/B8. (The negative staining phenomenon in the urine sample is caused by urinary albumin which prevents immobilization of heparan sulphate proteoglycan on the nylon membrane; indicated by an arrow). For comparison, heparan sulphate proteoglycan isolated from human aorta (3) is given. 
these antibodies recognize epitopes present on the core protein of the small heparan sulphate proteoglycan.

Different approaches to establishing a sandwich immunoassay, using several of the monoclonal antibodies against the small heparan sulphate proteoglycan, failed. This was most probably due to the fact that the antibodies used were specific for the relatively small core protein $\left(M_{\mathrm{r}} 22000-24000\right)$. As a sandwich assay depends on either repetitive epitopes or at least expression of two different epitopes on the antigen, steric hindrance might have occurred. Therefore, in a second attempt we have tried to establish a competitive antigen inhibition assay, since this type of assay does not depend on the expression of repetitive epitopes or at least two different epitopes. In this type of assay a constant amount of biotin-labelled antigen is added to the sample, and competes with the unlabelled antigen present in the sample for binding to an immobilized antibody. Biotinlabelled antigen bound to the antibody can then be detected by the use of streptavidin-peroxidase. However, this approach was also unsuccessful. A main reason was that biotinylation of the antigen seemed to alter the binding affinity of the antibody.

Therefore, we finally decided to establish an antigen binding assay in which the strongly negatively charged polyanionic heparan sulphate proteoglycan is bound to the surface of a cationic charge modified 96-well microtitre plate. Bound antigen can then be detected by the specific monoclonal antibody 1F10/B8 and a peroxidase-conjugated goat-anti-mouse-Ig antibody. This approach was successful. An example of the standard binding curve of this assay is shown in figure 2. Heparan

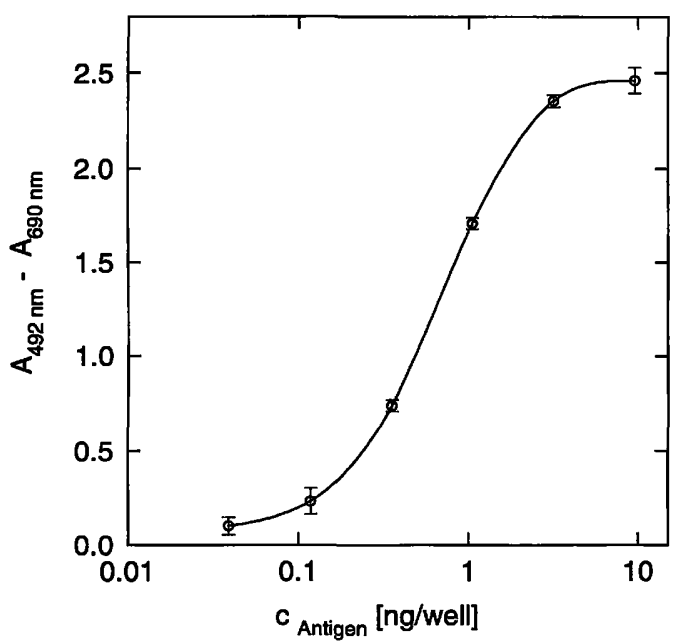

Fig. 2 Standard binding curve obtained with the monoclonal antibody 1 F10/B8

Increasing amounts of small basement membrane associated heparan sulphate proteoglycan $(0.032$ to $9.6 \mathrm{ng} /$ well $)$ were allowed to bind for $1 \mathrm{~h}$ to the cationized surface of the microtitre plate. Bound heparan sulphate proteoglycan was detected using a second peroxidase-conjugated polyclonal goat-anti-mouse-Ig antiserum as described under Material and Methods. sulphate proteoglycan concentrations in the range of $0.32-9.6 \mathrm{ng} /$ well can be measured, corresponding to a concentration of $32-9600 \mu \mathrm{g} / 1$ in the samples. Non-specific binding was successfully overcome in this assay by using $0.5 \mathrm{~g} / 1$ Tween- 20 in the sample buffer. No inhibition of binding was observed in the assay when other polyanionic molecules such as heparin or keratan sulphate (up to $5 \mathrm{mg} / \mathrm{l}$ heparin and $25 \mathrm{mg} / \mathrm{l} \mathrm{keratan}$ sulphate) were added (data not shown). The influence of excreted proteins, which frequently occurs in proteinuria, was analyzed by the addition of albumin to the samples up to a concentration of $5 \mathrm{~g} / \mathrm{l}$. Up to this concentration of albumin no inhibition of heparan sulphate proteoglycan binding was observed at higher concentrations of heparan sulphate proteoglycan and only a minor inhibition $(<15 \%)$ was observed at concentrations between 3.2 and $1 \mathrm{ng} /$ well (data not shown). In patients with a proteinuria exceeding $5 \mathrm{~g} / \mathrm{l}$, the glomerular basement membrane is severely damaged with a greatly reduced content of heparan sulphate proteoglycan. In these patients analysis of the excretion of heparan sulphate proteoglycan will be of limited value.

The reliability of the assay was analyzed with respect to sensitivity and precision. For the within-run and between-run imprecision, coefficient of variation (CV) values of $\leqq 10 \%$ were obtained. The $\mathrm{CV}$ values were determined for heparan sulphate proteoglycan concentrations of $3.2 \mathrm{ng} /$ well $(\mathrm{n}=10$, within-run $\mathrm{CV}=4.1 \%$, between-run $\mathrm{CV}=5.6 \%$ ). Under the conditions described the lower detection limit of the assay was about $20 \mu \mathrm{g} / 1$.

For heparan sulphate proteoglycan excreted into urine, data were expressed either as $\mu \mathrm{g} / 1$ of heparan sulphate proteoglycan or as a heparan sulphate proteoglycan/creatinine excretion ratio $(\mathrm{mg} / \mathrm{mol}$ creatinine). The mean urinary heparan sulphate proteoglycan concentration in normal control subjects was $28 \pm 8 \mu \mathrm{g} / \mathrm{l}(\mathrm{n}=17)$. The mean urinary heparan sulphate proteoglycan/creatinine excretion ratio in normal control subjects was 3.74 $\pm 1.29 \mathrm{mg} / \mathrm{mol}$. The relatively broad range of individual heparan sulphate proteoglycan excretion rates might be due to the influence of exercise on the heparan sulphate proteoglycan/creatinine excretion ratio. In fact, preliminary data indicate that the heparan sulphate proteoglycan/creatinine excretion ratio shows a strong increase after exercise. Therefore, a standardized exercise protocol was developed, which allows the measurement of the short-term exercise-induced increased excretion of heparan sulphate proteoglycan under conditions where de novo synthesis of heparan sulphate proteoglycan could be excluded. Under these conditions, the exerciseinduced excretion of heparan sulphate proteoglycan correlated with the content of heparan sulphate proteoglycan, as evidenced by immuno-histochemistry (19). According to these data, heparan sulphate proteoglycan 
excretion in normal subjects most probably reflects turnover of heparan sulphate proteoglycan in the glomerular basement membrane. However, the molecular mechanism of the release of heparan sulphate proteoglycan from basement membranes remains to be clarified. Whereas in humans no data are available on the turnover of basement membrane heparan sulphate proteoglycans, results from an experimental rat model indicate that heparan sulphates in the kidney exhibit a very rapid turnover with a half-life in the range of 5-20 hours, when examined by metabolic labelling and immunoprecipitation (20-22). In contrast, for collagen from glomerular basement membrane of the rat, a half-life of about 110 days has been found (23).

\section{References}

1. Farquhar MG. The glomerular basement membrane: a selective macromolecular filter. In: Hay ED, editor. Cell biology of the extracellular matrix. 2nd ed. New York: Plenum Press, 1991:367-418.

2. Timpl R. Structure and biological activity of basement membrane proteins. Eur J Biochem 1989; 180:487-503.

3. Kanwar YS, Liu ZZ, Kashihara N, Wallner EI. Current status of the structural basis of glomerular filtration and proteinuria. Sem Nephrol 1991; 11:390-413.

4. Venkatachalam MA, Rennke HG. The structural and molecular basis of glomerular filtration. Circulation Res 1978; 43:33749.

5. Bohrer MP, Baylis C, Humes D, Glassock RJ, Robertson CR, Brenner BM. Permselectivity of the glomerular capillary wall. J Clin Invest 1978; 61:72-8.

6. Moss J, Woodrow DF, Shore I, Gower P, Phillips M, Spiro RG. Ultrastructural immunogold studies of heparan sulphate proteoglycan in normal human glomeruli and glomerulonephritis. J Pathol 1990; 161:137-43.

7. Nakamura T, Ebihara I, Tomino Y, Koide H, Kikuchi K, Koiso $\mathrm{K}$. Gene expression of growth-related proteins and ECM constituents in response to unilateral nephrectomy. Am J Physiol 1992; 262:389-96.

8. Nerlich A, Schleicher ED. Immunohistochemical localisation of extracellular matrix components in human diabetic glomerular lesions. Am J Pathol 1991; 139:889-99.

9. Schneeberger EE, Stavrakis G, Mc Carthy K. Alternations in glomerular anionic sites in autologous immune complex nephritis. Lab Invest 1983; 49:445-52.

10. Makino H, Ideda S, Haramoto T, Ota Z. Heparan sulphate proteoglycans are lost in patients with diabetic nephrophathy. Nephron 1992; 61:415-21.

11. Stöcker G, Wilgenbus $\mathrm{K}$, Wagener $\mathrm{C}$, Greiling $\mathrm{H}$, Haubeck HD. Biochemical and immunochemical characterization of small basement membrane heparan sulphate proteoglycans from human aorta and kidney. J Cell Biol 1996; submitted for publication.

12. Stöcker G, Fischer DC. Isolation and characterization of proteoglycans from different tissues. J Chromat 1990; 521:31124.

13. Stöcker G, Lückge J, Greiling H, Wagener C. Characterization of biotin-labeled proteoglycans by electrophoretic separation on minigels and blotting onto nylon membranes prior and after enzymatic digestion. Anal Biochem 1989; 179:245-50.

14. Köhler G, Milstein C. Continuous culture of fused cells secreting antibodies of predefined specificity. Nature 1975; 256:495-7.

15. Iozzo R, Murdoch A. Proteoglycans of the extracellular environment: clues from the gene and protein offer novel perspec-
The assay described in the present report will allow the sensitive determination of basement membrane associated heparan sulphate proteoglycan levels in human urine and the detection of basement membrane alterations in the course of renal diseases. Therefore, development of this assay might be an important contribution to the spectrum of methods currently used for the analysis of proteinuria, such as the measurement of protein concentrations or analysis of the protein pattern in urine by SDS-PAGE (24-27). The novel enzyme immunoassay may be especially useful in the non-invasive analysis of basement membrane metabolism and breakdown in renal diseases, in monitoring disease progression and therapy control.

tives in molecular diversity and functions. FASEB J 1996; 10:598-614

16. Van den Born J, van den Heuvel LP, Bakker MA, Veerkamp $\mathrm{JH}$, Assmann $\mathrm{KJ}$, Berden $\mathrm{JH}$. Production and characterization of a monoclonal antibody against human glomerular heparan sulphate. Lab Invest 1991; 65:287-97.

17. Thomas GJ, Jenner L, Mason RM, Davies M. Human glomerular epithelial cell proteoglycans. Arch Biochem Biophys 1990; 278:11-20.

18. Van den Heuvel LP, van den Born J, van de Velden TJ, Veerkamp JH, Monnens LA, Schroder $\mathrm{CH}$, et al. Isolation and partial characterization of heparan sulphate proteoglycan from the human glomerular basement membrane. Biochem J 1989; $264: 457-65$.

19. Heintz B, Stöcker G, Mrowka C, Rentz U, Melzer H, Stickeler E, et al. Decreased glomerular basement membrane heparan sulphate proteoglycan in essential hypertension. Hypertension 1995; 25:399-407.

20. Hascall VC, Heinegard DK, Wight TN. Proteoglycans: metabolism and pathology. In: Hay ED, editor. Cell biology of the extracellular matrix. 2nd ed. New York: Plenum Press, 1991; $149-75$.

21. Beavan LA, Davies M, Couchman JR, Williams, MA, Mason $\mathrm{RM}$. In vivo turnover of the basement membrane and other heparan sulphate proteoglycans of rat glomerulus. Arch Biochem Biophys 1989; 269:576-85.

22. Cohen MP, Surma ML. 35-S-sulphate incorporation into glomerular basement membrane glycosaminoglycans is decreased in experimental diabetes. J Lab Clin Med 1981; 98:715-22.

23. Price RG, Spiro RG. Studies on the metabolism of the renal glomerular basement membrane. J Biol Chem 1977; 252:8597-602.

24. Houser MT. Characterization of proteinuria using random urine samples. Int J Pediatr Nephrol 1986; 7:197-202.

25. Ginsberg JM, Chang BS, Matarese RA, Garella S. Use of single voided urine samples to estimate quantitative proteinuria. N Engl J Med 1983; 309:1543-46.

26. Hofmann W, Rossmüller B, Guder WG, Edel H. A new strategy for characterizing proteinuria and haematuria from a single pattern of defined proteins in urine. Eur $\mathrm{J}$ Clin Chem Clin Biochem 1992; 30:707-12.

27. Hofmann W, Regenbogen C, Edel H, Guder WG. Diagnostic strategies in urine analysis. Kidney Int Suppl 1994; 47:111-4.

\section{Received October 15, 1996}

Corresponding author: PD. Dr. H.-D. Haubeck, Institut für Klinische Chemie und Pathobiochemie, RWTH Aachen, Pauwelsstraße 30, D-52057 Aachen, Germany 
